\title{
Scaling laws of resistive magnetohydrodynamic reconnection in the high-Lundquist-number, plasmoid-unstable regime
}

\author{
Yi-Min Huang $1,2,3, *$ and A. Bhattacharjee ${ }^{1,2,3,+}$ \\ ${ }^{1}$ Space Science Center, University of New Hampshire, Durham, NH 03824 \\ ${ }^{2}$ Center for Integrated Computation and Analysis of Reconnection and Turbulence \\ ${ }^{3}$ Center for Magnetic Self-Organization in Laboratory and Astrophysical Plasmas
}

\begin{abstract}
The Sweet-Parker layer in a system that exceeds a critical value of the Lundquist number $(S)$ is unstable to the plasmoid instability. In this paper, a numerical scaling study has been done with an island coalescing system driven by a low level of random noise. In the early stage, a primary Sweet-Parker layer forms between the two coalescing islands. The primary Sweet-Parker layer breaks into multiple plasmoids and even thinner current sheets through multiple levels of cascading if the Lundquist number is greater than a critical value $S_{c} \simeq 4 \times 10^{4}$. As a result of the plasmoid instability, the system realizes a fast nonlinear reconnection rate that is nearly independent of $S$, and is only weakly dependent on the level of noise. The number of plasmoids in the linear regime is found to scales as $S^{3 / 8}$, as predicted by an earlier asymptotic analysis (Loureiro et al., Phys. Plasmas 14, 100703 (2007)). In the nonlinear regime, the number of plasmoids follows a steeper scaling, and is proportional to $S$. The thickness and length of current sheets are found to scale as $S^{-1}$, and the local current densities of current sheets scale as $S^{-1}$. Heuristic arguments are given in support of theses scaling relations.
\end{abstract}

*Electronic address: yimin.huang@unh.edu

${ }^{\dagger}$ Electronic address: amitava.bhattacharjee@unh.edu 


\section{INTRODUCTION}

Recent studies of nonlinear reconnection in large high-Lundquist-number $(S)$ plasmas, based on resistive magnetohydrodynamics (MHD) [1] as well as fully kinetic simulations that include a collision operator [2] have produced a surprise. It is seen in these studies that above a critical value of the Lundquist number, the system deviates qualitatively from the predictions of Sweet-Parker theory [3, 4] which has been the standard model for reconnection in the high- $S$ regime. In the Sweet-Parker model, the reconnection layer has the structure of Y-points, with a length of the order of the system size, and a width given by $\delta_{S P}=$ $L / S^{1 / 2}$, where $S=L V_{A} / \eta$ is the Lundquist number based on the system size $L$, the Alfvén speed $V_{A}$, and the magnetic diffusivity $\eta$. The Sweet-Parker model is usually considered to be a model of "slow" reconnection as it predicts the reconnection rate to scale as $S^{-1 / 2}$. (The Petschek model [5] predicts a much weaker dependence on $S$, with the maximum reconnection rate $\sim 1 / \log S$. However, it has become clear over the years that Petschek model is realizable only when the resistivity is locally enhanced around the reconnection site. [6]) For weakly collisional systems such as the solar corona, the Lundquist number is typically very large $\left(\sim 10^{12}-10^{14}\right)$ and the Sweet-Parker reconnection time scale is of the order of years, much too slow to account for fast events such as solar flares. The SweetParker model is based on the assumption of the existence of a long thin current layer. Although it has been known for some time that such a thin current layer may be unstable to a secondary tearing instability (referred to hereafter as the plasmoid instability) which generates plasmoids, [7, $8,19,10,11,12]$ it has been realized only fairly recently that the Sweet-Parker layer actually becomes more unstable as the Lundquist number increases, with a linear growth rate $\gamma \sim S^{1 / 4}$ and the number of plasmoids $\sim S^{3 / 8}$. [1, 13] In a recent paper

(Ref. [1], hereafter referred to as Paper I), Bhattacharjee et al. have presented numerical results that suggest strongly that as a consequence of the plasmoid instability, the system evolves into a nonlinear regime in which the reconnection rate becomes weakly dependent on $S$.

The primary goal of this paper is to strengthen the results obtained in Paper I in two significant ways: first, to present new simulation results with a modified initial condition that enables us to obtain stronger scaling results on the nonlinear reconnection rate and the number of plasmoids generated in the nonlinear regime, and second, a simple heuristic 
model that is consistent with the results of the simulation and fortifies the claim in Paper I that the reconnection rate in the nonlinear regime of the plasmoid instability is fast and independent of $S$.

\section{NUMERICAL MODEL}

The initial condition in Paper I does not have a thin current sheet to begin with. It has four magnetic islands and is unstable to an ideal coalescence instability. After the onset of the coalescence instability, a Sweet-Parker current sheet is created when two islands are attracted toward each other. 14] In this case, there is a relatively long initial transient period before the reconnection process starts. Furthermore, the dynamics of the system are complicated by the sloshing of coalescing islands that causes the primary Sweet-Parker layer to lengthen or shorten from time to time. This makes it difficult to verify the predictions of linear theory in this particular system. The present study seeks remedies to these two drawbacks. We still consider the merging of two islands, but now put them in close contact initially. There is an initial current layer between the flux tubes, which quickly (typically within less than one Alfvén time) adjusts its width depending on the Lundquist number to form the primary Sweet-Parker layer, which may subsequently become unstable to the plasmoid instability. In this new system, the transient period is significantly shortened and the sloshing between islands is largely eliminated. It is still not easy to measure the linear growth rate in this new model, but we can at least verify the scaling of the number of plasmoids in the linear regime.

The initial condition is similar to the model of Uzdensky and Kulsrud. 15] To start with, let $\psi_{0}=\cos (\pi x) \sin (2 \pi|z|) / 2 \pi$ and $\mathbf{B}_{0}=\nabla \psi_{0} \times \hat{\mathbf{y}}$ in the domain $(x, z) \in[-1 / 2,1 / 2] \times$ $[-1 / 2,1 / 2]$. The $\psi_{0}$ so defined satisfies $\nabla^{2} \psi_{0}=-5 \pi^{2} \psi_{0}$. If the pressure is set to $p_{0}=$ $C+5 \pi^{2} \psi_{0}^{2} / 2$, with $C$ an arbitrary constant, the system is in force balance. However, the magnetic field defined by $\psi_{0}$ has a tangential discontinuity at $z=0$, which causes numerical difficulties. We smooth it out as $\psi=\tanh (\alpha z) \cos (\pi x) \sin (2 \pi z) / 2 \pi$, where $\alpha$ is a large number. This smoothed function $\psi$ no longer satisfies $\nabla^{2} \psi=f(\psi)$ and the magnetic force cannot be balanced entirely by pressure. However, the magnetic force can be canceled to a large extent if the pressure is set to $p=p_{0}+\left(B_{x 0}^{2}-B_{x}^{2}\right) / 2$, where $B_{x 0}=-\partial_{z} \psi_{0}$ and $B_{x}=-\partial_{z} \psi$, respectively. We assume the isothermal equation of state, $p=2 \rho T$, in our 
simulation. We choose $T=3, C=2 T$, and $\alpha=100$. For these parameters, the initial plasma density varies from 0.96 to 1.1 and the plasma beta $\left(\beta \equiv p / B^{2}\right)$ obeys the inequality $\beta \gtrsim 6$. The system is, therefore, nearly incompressible. Figure 1 shows the initial current density and magnetic field lines.

We find in the present study that the plasmoid instability depends on the noise level of the system, at least when the Lundquist number is not far above the critical value. Due to the outflow in the primary Sweet-Parker layer, if the noise level is low, the plasmoid instability may not grow to visible size before being convected out. When we seed the system initially with random noise, at relatively low values of the Lundquist number $\left(\sim 10^{5}\right)$ we obtain a short burst of plasmoids, following which the current layer becomes stable again after all the plasmoids are convected out. Because of this, we have included a random forcing in the system, which enables us also to study the effect of noise level on the reconnection rate. (Notice that we did not apply any random forcing or noise in Paper I. The sloshing between coalescing islands is a natural source of noise, but uncontrolled.) The governing equations for the time evolution of the system are:

$$
\begin{gathered}
\partial_{t} \rho+\nabla \cdot(\rho \mathbf{v})=0 \\
\partial_{t}(\rho \mathbf{v})+\nabla \cdot(\rho \mathbf{v v})=-\nabla p-\nabla \psi \nabla^{2} \psi+\epsilon \mathbf{f}(\mathbf{x}, t), \\
\partial_{t} \psi+\mathbf{v} \cdot \nabla \psi=\eta \nabla^{2} \psi,
\end{gathered}
$$

where a random forcing term $\epsilon \mathbf{f}(\mathbf{x}, t)$ is added to the right hand side of the momentum equation (2). The forcing function is white noise in both space and time with $\langle\mathbf{f}\rangle=0$, and $\epsilon$ is a small parameter which controls the noise level. By white noise we mean that $\left\langle f_{i}(\mathbf{x}, t) f_{j}\left(\mathbf{x}^{\prime}, t^{\prime}\right)\right\rangle \sim \delta_{i j} \delta\left(\mathbf{x}-\mathbf{x}^{\prime}\right) \delta\left(t-t^{\prime}\right)$, where $i$ and $j$ can be $x$ or $z$ and \langle\rangle is the ensemble average. Care has to be taken to ensure that the discrete representation is independent of the time step. Our implementation is similar to that in Ref.[16]. It is convenient to set $\mathbf{f}=\rho \mathbf{a}$, where $\mathbf{a}$ is a random acceleration. In a single time step, the momentum density evolves from $\rho \mathbf{v}$ to $\rho \mathbf{v}+\epsilon \rho \mathbf{a} \Delta t$ (neglecting other forces), and the kinetic energy density evolves from $\rho v^{2} / 2$ to $\rho v^{2} / 2+\epsilon \rho \mathbf{a} \cdot \mathbf{v} \Delta t+\epsilon^{2} \rho a^{2} \Delta t^{2} / 2$. Therefore, the average power density from the random force is

$$
\frac{\left\langle\epsilon \rho \mathbf{a} \cdot \mathbf{v} \Delta t+\epsilon^{2} \rho a^{2} \Delta t^{2} / 2\right\rangle}{\Delta t}=\frac{1}{2} \epsilon^{2} \rho \Delta t\left\langle a^{2}\right\rangle,
$$


where $\langle\mathbf{a} \cdot \mathbf{v}\rangle=0$ is used, and $a^{2}=\mathbf{a} \cdot \mathbf{a}$. At each grid point, we set $a_{x}, a_{z}$ to random numbers between -1 and 1 with a uniform probability distribution, divided by $\sqrt{\Delta t}$. That is,

$$
a_{i}=\frac{\operatorname{rand}(-1,1)}{\sqrt{\Delta t}} .
$$

Then $\Delta t\left\langle a^{2}\right\rangle=2 / 3$ and is independent of $\Delta t$. The average power density is $\epsilon^{2} \rho / 3$ and the total power $\epsilon^{2} M / 3$, with $M$ the total mass ( $M \simeq 1$ in our simulation). We use $\epsilon=10^{-5}-10^{-3}$ in our simulations and the corresponding power density ranges from $3 \times 10^{-11}$ to $3 \times 10^{-7}$. Since our simulations typically last only a few Alfvén times, the energy input from random forcing is negligible compared to the total magnetic energy $(\sim O(1))$ in the system. This ensures that the random forcing only provides noise for the instability to grow but does not otherwise alter the system in a significant way (see more discussion in Sec. III).

Our numerical algorithm [17] uses finite differences with a five-point stencil in each direction, and a second-order accurate trapezoidal leapfrog method for time stepping. We use a uniform grid along the $x$ direction, and a nonuniform grid in the $z$ direction that packs high resolution around $z=0$ in order to resolve the sharp spatial gradients in the reconnection layer. Perfectly conducting $\left(\partial_{t} \psi=0\right)$, impenetrable $(\mathbf{v} \cdot \hat{\mathbf{n}}=0)$, and free slipping $(\hat{\mathbf{n}} \cdot \nabla(\hat{\mathbf{n}} \times \mathbf{v})=0)$ boundary conditions are assumed ( $\hat{\mathbf{n}}$ is the unit normal vector to the boundary). We further assume reflection symmetry along the $x$ axis and only the region $z \geq 0$ is simulated. The highest resolution is 12800 in $x$ and 1600 in $z$, with the smallest grid size $\Delta z=5.4 \times 10^{-6}$.

\section{NUMERICAL RESULTS}

One of the key objectives of this study is to determine the scaling of reconnection rate in the plasmoid-unstable regime. To quantify the speed of reconnection, we measure the time it takes to reconnect a significant portion of the magnetic flux within the two merging islands. The amount of magnetic flux in an island is $\psi_{\max }-\psi_{s}$, where $\psi_{\max }$ is the maximum of $\psi$ in the island and $\psi_{s}$ is the value of $\psi$ at the separatrix separating the two merging islands. Initially $\psi_{\max } \simeq 0.16$ and it remains approximately unchanged since the resistivity is low; therefore it suffices to just measure $\psi_{s}$. We denote the time it takes to reconnect from $\psi_{s}=0.01$ to $\psi_{s}=0.05$ as $t_{r e c}$. The starting point $\psi_{s}=0.01$ is chosen to allow the plasmoid instability to build up, and the end point $\psi_{s}=0.05$ is chosen such that the reconnection 
layer does not shorten too much compared with that in the initial condition. The range corresponds to reconnecting $25 \%$ of the initial flux.

Figure 2 shows the reconnection time $t_{\text {rec }}$ for various $S$ and $\epsilon$. For lower $S$, the reconnection time $t_{r e c} \sim S^{1 / 2}$, as expected from the Sweet-Parker theory. [15] The critical Lundquist number for plasmoid instability is about $S_{c} \simeq 4 \times 10^{4}$. Above $S_{c}$, the reconnection time $t_{r e c}$ deviates from the Sweet-Parker scaling and becomes shorter. In the plasmoid unstable regime, the reconnection time is nearly independent of $S$. However, the reconnection time has a weak dependence on the noise level throughout the $S$ range we have tested. The plateaued values of $t_{\text {rec }}$ in the high- $S$ regimes for $\epsilon=10^{-3}, 10^{-4}$, and $10^{-5}$ are $5.30 \pm 0.27$, $6.10 \pm 0.41$, and $7.05 \pm 0.16$, respectively. Here we take the average values over the range $S=5 \times 10^{5}$ to $3 \times 10^{6}$, and the errors represent the standard deviation. We have tested the convergence of our numerical results by varying the resolution, the time step, and the random seed for selected runs. These are represented by multiple data points with the same parameters in Figure 2. The results are fairly consistent, with fluctuations no more than a few percent. The dependence of $t_{r e c}$ on $\epsilon$ may be tentatively fit with a power law, which gives $t_{\text {rec }} \sim \epsilon^{-0.062}$. However, given the limited range of the parameter space we have explored, this scaling should not be considered as conclusive.

The global characteristic values for $V_{A}$ and $B$ are about 1 , which yield the normalized average reconnection rate as

$$
\frac{1}{B V_{A}}\left\langle\frac{d \psi_{s}}{d t}\right\rangle=\frac{0.04}{t_{\text {rec }}}
$$

In the high Lundquist number regime, $t_{\text {rec }} \simeq 5$ to 7 from our simulations and the normalized reconnection rate is in the range 0.006 to 0.008 . The normalized reconnection rate obtained here is similar to the result of Paper I.

Figure 3 shows a time sequence of the current density, overlaid with magnetic field lines, within a small area $(1 / 1000)$ of the whole domain for a case with $\epsilon=10^{-3}$ and $S=3 \times 10^{6}$. The initial current layer (panel (a)) quickly thins down to form the primary Sweet-Parker layer (panel(b)), which becomes unstable to the plasmoid instability (panel (c)). As the instability proceeds, the plasmoids grow in size and the current sheets between plasmoids are again Sweet-Parker like (panel (d)). These secondary Sweet-Parker current sheets are thinner than the primary one and are again unstable to the tertiary plasmoid instability (panel (e)). This process of multiple stages of cascading resembles the scenario envisaged in Ref.[11]. The plasmoids can merge to form larger ones and new plasmoids are constantly 
generated (panel (f)). Figure 4 shows the global configuration at a later time $t=3.9$, as well as close-ups of the reconnection layer. The figure shows that on a large, coarse-grained scale, the configuration looks Sweet-Parker like, except for the important difference that the reconnection layer is no longer a single extended current sheet, but is made up of a sequence of copious plasmoids and current sheets.

Linear theory predicts that the number of plasmoids, $n_{p}^{L}$, scales as $S^{3 / 8}$. [1, 13] We verify this by counting the maximum number of plasmoids within the central region, $-0.25 \leq x \leq$ 0.25, before the plasmoid instability becomes highly nonlinear (roughly corresponds to panel (c) in Figure 31). Figure 5 shows the number of plasmoids versus $S$, for $\epsilon=10^{-3}$, in both linear and nonlinear (see the discussion later) regimes. The result in the linear regime is in good agreement with the $S^{3 / 8}$ scaling predicted by asymptotic analysis. This scaling has been verified by Samtaney et al. with local simulations up to $S=10^{8}$. [18]

In the fully nonlinear regime, the plasmoid dynamics are very complicated and constantly evolving. Plasmoids may grow in size, coalesce with each other to form larger plasmoids, and finally get ejected into the downstream region. Meanwhile, new plasmoids are constantly generated in the reconnection layer. We may regard the reconnection layer with multiple plasmoids as a statistical steady state. As a simple, first approximation, we expect the cascading to stop when the current sheet segments between plasmoids become stable. We may imagine the reconnection layer as a chain of plasmoids connected by marginally stable Sweet-Parker current sheets. For given $\eta$ and $V_{A}$, the critical length of a marginally stable current layer is $L_{c}=S_{c} \eta / V_{A}$. Therefore we expect the number of plasmoids in the nonlinear regime, $n_{p}^{N L}$, to scale like $L / L_{c} \sim S / S_{c}$. Furthermore, the thickness of each Sweet-Parker sheet is $\delta_{c} \sim L_{c} / \sqrt{S_{c}} \sim \eta \sqrt{S_{c}} / V_{A} \sim \delta_{S P} \sqrt{S_{c} / S}$, and the current density $J \sim B / \delta_{c} \sim B V_{A} / \eta \sqrt{S_{c}} \sim B S / L \sqrt{S_{c}}$. If we identify the reconnection rate with $\eta J$, then the reconnection rate $\sim \eta J \sim B V_{A} / \sqrt{S_{c}}$, which is independent of $S$. This is consistent with our finding that the reconnection rate is nearly independent of $S$ in the high- $S$ regime. Clearly, the assumption that all current sheets are marginally stable, and therefore all identical, is oversimplified. If we look at the individual current sheets, there are a whole variety of them, each with a different length, width, and current density. Therefore, the system is better described with a statistical approach. If we neglect complications such as asymmetry or background shear flow, and consider the simple Sweet-Parker picture for each current sheet, then the local Lundquist number $S_{l o c a l} \equiv V_{A} l / \eta$ is the only dimensionless parameter 
associated with it. Here $l$ denotes the length of the current sheet. The current sheet thickness will be $\delta \sim l / \sqrt{S_{\text {local }}}$, as predicted by the Sweet-Parker theory. The local Lundquist number being greater or smaller than $S_{c}$ determines whether a current sheet may or may not further break into plasmoids and even smaller current sheets. Because it is the local Lundquist number that determines the cascading of a local current sheet to even smaller scales, we hypothesize that the probability distribution of $S_{\text {local }}$ is independent of the global Lundquist number. The underlying assumption is that, if we consider the ensemble of local current sheets and characterize each current sheet by a dimensionless parameter $S_{\text {local }}$, there is a similarity across systems of different global Lundquist numbers. If we further assume that the local upstream Alfvén speed is determined by global conditions, it follows that statistically the length $l$ and the thickness $\delta$ of a current sheet scale as $\eta$, and the current density $J$ scales as $\eta^{-1}$. If we consider the simple picture that two neighboring current sheets are separated by a plasmoid, then $l \sim \eta$ implies the number of plasmoids in the nonlinear regime $\sim \eta^{-1}$.

Now we proceed to examine whether the conclusions from the simple heuristic argument are consistent with our simulation data. Here we present the results from cases with $\epsilon=10^{-3}$. Results from other values of $\epsilon$ are similar. We count the number of plasmoids by first identifying X-points and O-points along $z=0$. There are two types of O-points, the local minimum (type I) and the local maximum (type II) of $\psi$. Likewise, there are two types of X-points, one with $\partial_{x}^{2} \psi<0, \partial_{z}^{2} \psi>0$ (type I) and the other with $\partial_{x}^{2} \psi>0, \partial_{z}^{2} \psi<0$ (type II). In the linear regime, only X-points and O-points of type I are present. When plasmoids start to coalesce with each other, type II null points may be created. We count the number of type I O-points within $-0.25 \leq x \leq 0.25$ as the number of plasmoids in the nonlinear regime. As shown in Figure 5, the number of plasmoids in the nonlinear regime appears to agree with the $\sim S$ scaling. Because the number of plasmoids fluctuates, the median value is used; the error bar indicates the range between the first and the third quartiles. Notice that although the number of plasmoids in the nonlinear regime follows a steeper scaling than that in the linear regime, it is not until about $S=2 \times 10^{6}$ that the nonlinear scaling catches up with the linear counterpart. This is because at lower $S$, the coalescence and ejection of plasmoids exceeds the generation of new plasmoids. Equating the estimate, $n_{p}^{L} \simeq S^{3 / 8} / 2 \pi$, from the linear theory [1, 13] with the heuristic nonlinear estimate, $n_{p}^{N L} \simeq S / S_{c}$, and using $S_{c} \simeq 4 \times 10^{4}$, we obtain $n_{p}^{L} \simeq n_{p}^{N L}$ when $S \simeq 1.2 \times 10^{6}$, which is in approximate agreement 
with the observed $S=2 \times 10^{6}$.

To examine the statistics of current sheets, we have to first set up a diagnostic for a current sheet, which is subject to a certain degree of arbitrariness. We search for local maxima of $J$ greater than $10 \%$ of the global maximum $J_{\max }$ within $-0.25 \leq x \leq 0.25$ as potential sites of current sheets. However, two neighboring maxima are regarded as separate current sheets only when the trough between them is lower than $25 \%$ of the greater of the two. The length $l$ and the thickness $\delta$ of a current sheet are measured by the locations where the current density drops to $25 \%$ of the local maximum $J$ of the current sheet.

Figures 6 and 7 show scalings of the thickness $\delta$, length $l$, and current density $J$ with respect to the global Lundquist number $S$. The data are collected from time slices during the period to reconnect $25 \%$ of the flux in each case. Again the median values are used, and error bars indicate the range between the first and the third quartiles. Also shown for reference are the predictions from the heuristic argument based on marginally stable current sheets, i.e., $l \sim L_{c} \sim L S_{c} / S, \delta \sim \delta_{S P}\left(S_{c} / S\right)^{1 / 2} \sim L S_{c}^{1 / 2} / S$, and $J \sim B / \delta \sim B S / L S_{c}^{1 / 2}$. It is evident that the characteristics of current sheets are distributed over a broad range, as indicated by the rather large error bars. Clearly, the observed quantities follow the expected scalings. Quite surprisingly, the predictions for $\delta$ and $J$ from the heuristic argument are in good agreement with the observed median values, even though the argument itself is rather crude. However, the prediction of $l$ appears to be systematically an overestimate, and lies at the larger end of the numerically observed lengths. This is consistent with the fact that $L_{c}=L S_{c} / S$ is the critical length just above which the plasmoid instability is triggered. One may wonder how the prediction of $\delta$ can be consistent with the observed values when the prediction of $l$ is an overestimate. A possible explanation is that, the heuristic argument assumes Sweet-Parker-like local current sheets, but clearly not all current sheets in simulations are Sweet-Parker-like. The existence of non-Sweet-Parker-like current sheets is evident from the movie available online, which is for the case $S=3 \times 10^{6}, \epsilon=10^{-3}$.

Let us now take a more detailed look into the statistics of current sheets. Figure 8 shows the probability distribution functions (PDFs) of $\eta J$ for $S=10^{6}, 2 \times 10^{6}, 3 \times 10^{6}$, from cases with $\epsilon=10^{-3}$. The case $S=10^{6}$ has been done with two runs. The PDFs of $\eta J$ from different runs clearly show a degree of similarity, which lends some support to our hypothesis of similarity across systems of different global Lundquist number. However, we also notice some differences between the PDFs from different runs. Even the two runs with $S=10^{6}$ 
show a significant variation in the PDFs. Therefore, more study is needed to further assess the validity of our hypothesis. Ideally the same global setting should be repeated many times with different random seeds for better statistics, but that is computationally too expensive to be done at the present time.

Before we conclude this Section, we remark on a few subtle issues. In the heuristic argument given above, we have used the quantity $\eta J$ to estimate the reconnection rate. Strictly speaking, this is valid when the X-point and the stagnation point of the flow coincide, which is not necessarily the case when the reconnection layer is embedded with multiple Xpoints and plasmoids. Notwithstanding this caveat, we generally find that the peak value of $\eta J$ is a reasonable measure of the reconnection rate.

We also address the issue of whether random forcing, by itself, can significantly enhance the reconnection rate. An estimate of the effect of reconnection rate due to random fluctuations is the quantity $|\tilde{\mathbf{v}} \times \mathbf{B}|$ at the reconnection layer, where $\tilde{\mathbf{v}}$ is the random velocity fluctuation. Our estimates indicate that the contribution of random fluctuations is less than $1 \%$ of the observed reconnection rate in the high-S regime. This conclusion is reinforced by the fact that in the plasmoid stable regime, the variations in $t_{r e c}$ for different $\epsilon$ are negligible. This is qualitatively different from a recent turbulent magnetic reconnection study by Loureiro et al.,[19] where the system is more strongly driven, and the reconnection rate shows a noticeable dependence on the magnitude of the forcing even for a Lundquist number as low as $10^{3}$.

\section{SUMMARY AND CONCLUSION}

In summary, we have shown through a series of simulations that resistive MHD can achieve a fast reconnection rate in the high-Lundquist-number regime. Fast reconnection is facilitated by the plasmoid instability. The resultant reconnection rate is independent of $S$ and is weakly dependent on the noise level. We have verified the $S^{3 / 8}$ scaling of the number of plasmoids in the linear regime, as predicted in Refs. [1, 13]. In the nonlinear regime, the number of plasmoids follows a steeper scaling and is proportional to $S$. We also have done statistical studies of the local current sheets, and found that the current sheet thickness and length both scale as $S^{-1}$, while the current density scales as $S$. These findings are consistent with our heuristic argument and the claim that the reconnection rate is independent of $S$ 
in the high- $S$ regime.

The fast reconnection rate we have obtained is approximately $0.01 V_{A} B$, which is similar

to the values from other recent resistive MHD studies, [1, 20] but is smaller than the typical reconnection rate from collisionless two-fluid or particle-in-cell simulations by an order of magnitude. Which rate will be realized depends on how collisional the system is. If the Sweet-Parker thickness $\delta_{S P}$ is greater than the ion skin depth $d_{i}$ (or the ion Larmor radius at the sound speed, $\rho_{s}$, if there is a guide field) in a system, a Sweet-Parker layer is likely to form first. On the other hand, if $\delta_{S P}<d_{i}$ (or $\rho_{s}$ ), the reconnection would likely proceed dominated by collisionless effects. [21, 22, 23, 24, 25, 26] An interesting regime that has not drawn much attention before is when $\delta_{S P}>d_{i}$ (or $\rho_{s}$ ) but $S>S_{c}$. Then we expect the plasmoid instability to set in and the primary Sweet-Parker layer will break into segments. This brings the thickness further down to $\delta \sim \delta_{S P}\left(S_{c} / S\right)^{1 / 2}$. If $\delta>d_{i}$ (or $\left.\rho_{s}\right)$ then the system is still dominated by collisional effects and we may end up getting a reconnection rate of $0.01 V_{A} B$. However, if $\delta<d_{i}$ (or $\rho_{s}$ ) then the system will be in the collisionless regime. It is possible the reconnection rate will be further enhanced. Indeed, the recent particle-in-cell simulation by Daughton et al. suggests this possibility [2] but more needs to be done to determine if this is a general trend.

\section{Acknowledgments}

The authors would like to thank Dr. Brian P. Sullivan, Prof. Kai Germaschewski, Dr. William Fox, Dr. Hongang Yang, Prof. Barrett N. Rogers, and Prof. Chung-Sang Ng for beneficial conversations. We also acknowledge an anonymous referee for many constructive suggestions. This work is supported by the Department of Energy, Grant No. DE-FG0207ER46372, under the auspice of the Center for Integrated Computation and Analysis of Reconnection and Turbulence (CICART) and the National Science Foundation, Grant No. PHY-0215581 (PFC: Center for Magnetic Self-Organization in Laboratory and Astrophysical Plasmas). Computations were performed on facilities at National Energy Research Scientific Computing Center and the Zaphod Beowulf cluster, which was funded in part by the Major Research Instrumentation program of the National Science Foundation, Grant No. ATM- 
0424905 .

[1] A. Bhattacharjee, Y.-M. Huang, H. Yang, and B. Rogers, Phys. Plasmas 16, 112102 (2009).

[2] W. Daughton, V. Roytershteyn, B. J. Albright, H. Karimabadi, L. Yin, and K. J. Bowers, Phys. Rev. Lett. 103, 065004 (2009).

[3] P. A. Sweet, Nuovo Cimento Suppl. Ser. X 8, 188 (1958).

[4] E. N. Parker, Astrophys. J. Suppl. 8, 177 (1963).

[5] H. E. Petschek, in AAS/NASA Symposium on the Physics of Solar Flares, edited by W. N. Hess (NASA, Washington, DC, 1964), p. 425.

[6] D. Biskamp, Magnetic Reconnection in Plasmas (Cambridge University Press, 2000).

[7] S. V. Bulanov, J. Sakai, and S. I. Syrovatskii, Sov. J. Plasma Phys. 5, 157 (1979).

[8] L. C. Lee and Z. F. Fu, J. Geophys. Res. 91, 6807 (1986).

[9] D. Biskamp, Phys. Fluids 29, 1520 (1986).

[10] M. Yan, H. C. Lee, and E. R. Priest, Journal of Geophysical Research 97, 8277 (1992).

[11] K. Shibata and S. Tanuma, Earth Planets Space 53, 473 (2001).

[12] G. Lapenta, Phys. Rev. Lett. 100, 235001 (2008).

[13] N. F. Loureiro, A. A. Schekochihin, and S. C. Cowley, Phys. Plasmas 14, 100703 (2007).

[14] D. W. Longcope and H. R. Strauss, Phys. Fluids B 5, 2858 (1993).

[15] D. A. Uzdensky and R. M. Kulsrud, Phys. Plasmas 7, 4018 (2000).

[16] K. Alvelius, Phys. Fluids 11, 1880 (1999).

[17] P. N. Guzdar, J. F. Drake, D. McCarthy, A. B. Hassam, and C. S. Liu, Phys. Fluids B 5, 3712 (1993).

[18] R. Samtaney, N. F. Loureiro, D. A. Uzdensky, A. A. Schekochihin, and S. C. Cowley, PRL 103, 105004 (2009).

[19] N. F. Loureiro, D. A. Uzdensky, A. A. Schekochihin, S. C. Cowley, and T. A. Yousef, Mon. Not. R. Astron. Soc. 399, L146 (2009).

[20] P. A. Cassak, M. A. Shay, and J. F. Drake, Phys. Plasmas 16, 120702 (2009).

[21] A. Y. Aydemir, Phys Fluids B-Plasma Phys. 4, 3469 (1992).

[22] Z. W. Ma and A. Bhattacharjee, Geophys. Res. Lett. 23, 1673 (1996).

[23] J. C. Dorelli and J. Birn, J. Geophys. Res. 108, 1133 (2003). 


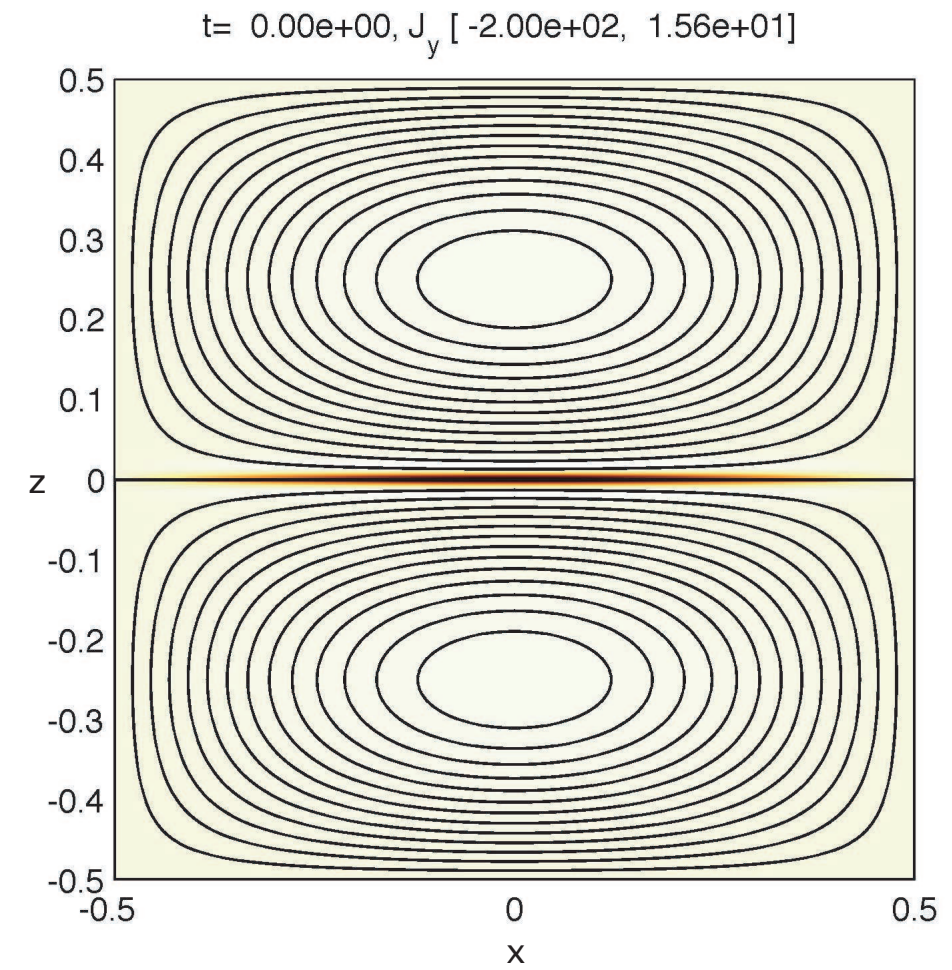

Figure 1: (Color online) The initial current density, with magnetic field lines (constant $\psi$ contours) overlaid.

[24] A. Bhattacharjee, Annu. Rev. Astron. Astrophys. 42, 365 (2004).

[25] P. A. Cassak, M. A. Shay, and J. F. Drake, Phys. Rev. Lett. 95, 235002 (2005).

[26] P. A. Cassak, J. F. Drake, and M. A. Shay, Phys. Plasmas 14, 054502 (2007). 


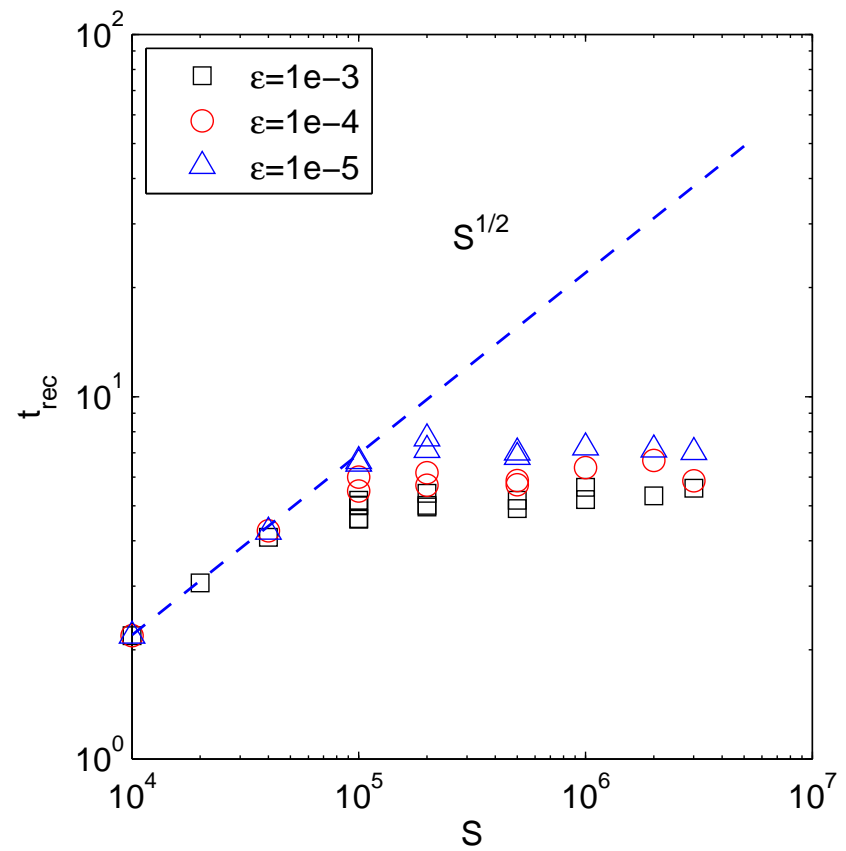

Figure 2: (Color online) The reconnection time $t_{r e c}$ for various $S$ and $\epsilon$. The dashed line is the Sweet-Parker scaling. 


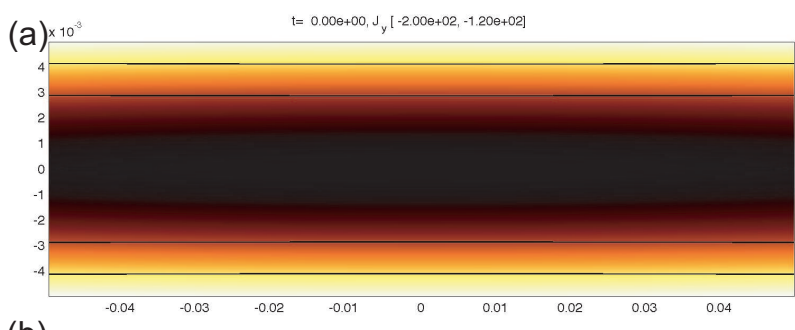

(b)

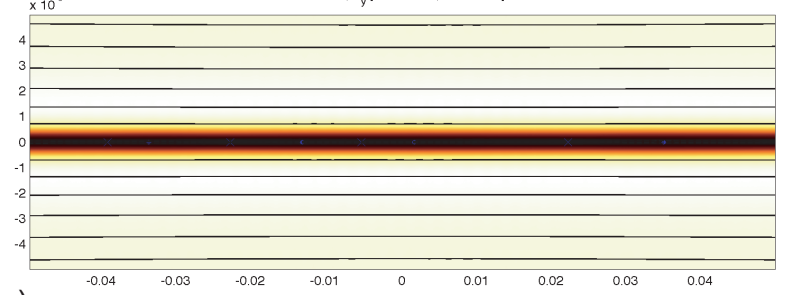

(c)

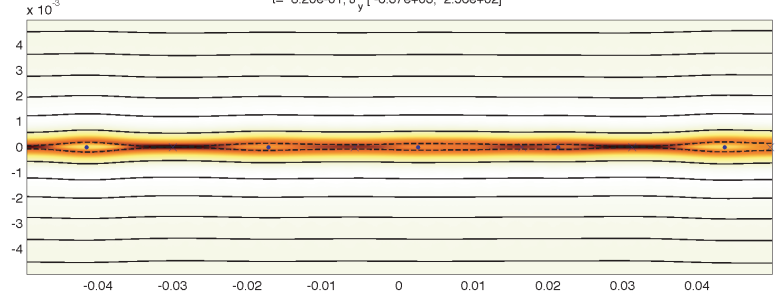

(d) $\times 10^{2}$

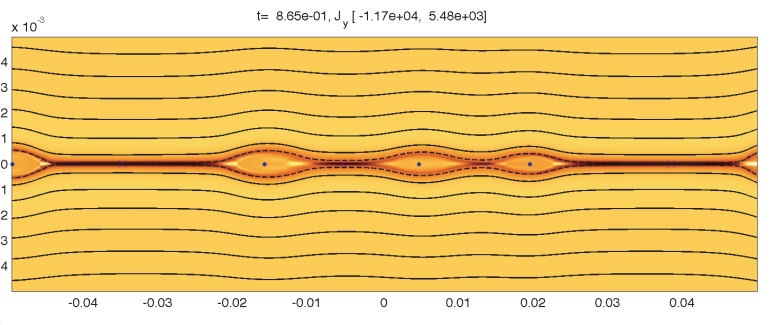

(e)
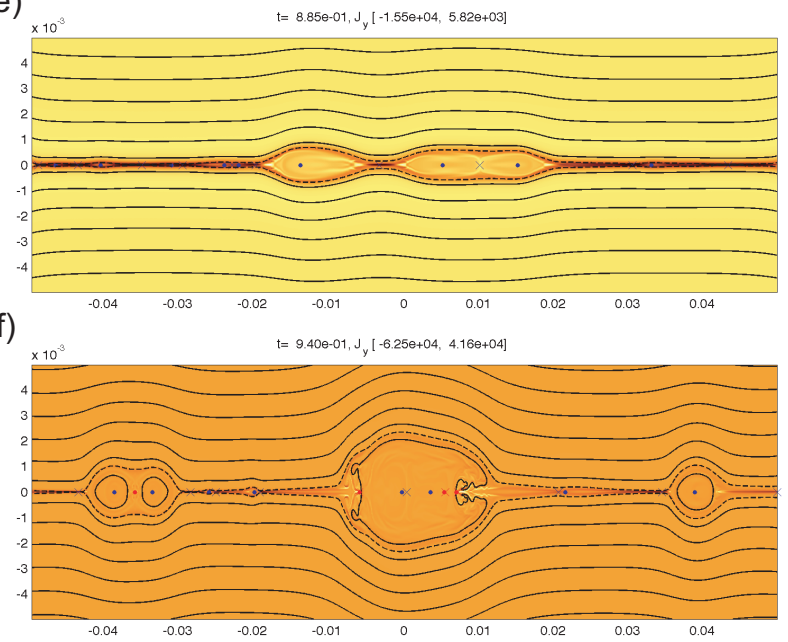

Figure 3: (Color online) Time sequence of the current density, overlaid with magnetic field lines, for the case $S=3 \times 10^{6}, \epsilon=10^{-3}$. The dashed line indicates the separatrix. The dots and crosses indicate $\mathrm{O}$ points and $\mathrm{X}$ points, respectively. The blue and the red colors indicate the two types of $\mathrm{X}$ and $\mathrm{O}$ points (blue for type I and red for type II). Notice that the vertical axis is stretched for better visualization. 
$t=3.90 e+00, J_{y}[-2.64 e+04,1.40 e+04]$
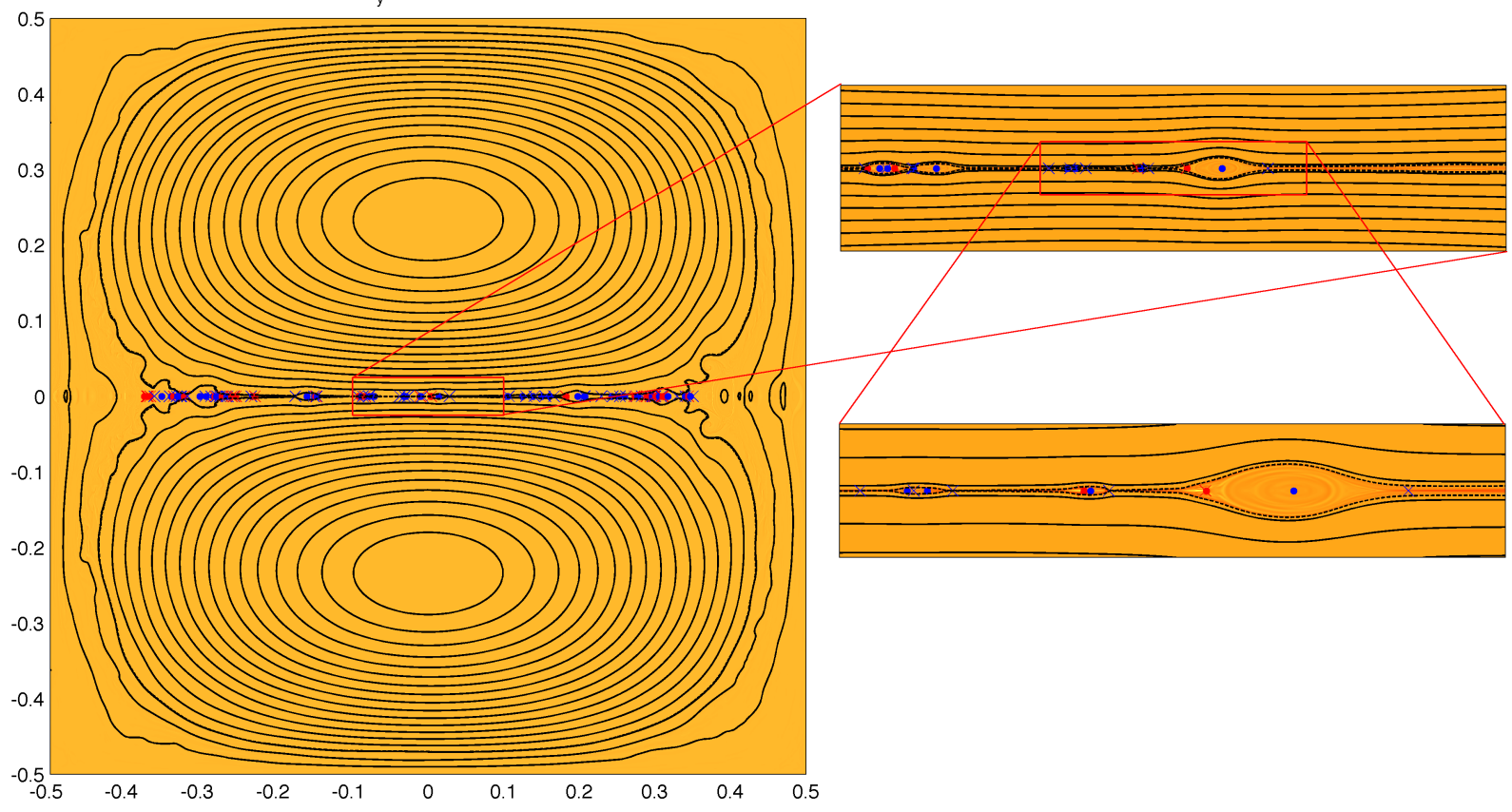

Figure 4: (Color online) The global configuration at a later time, $t=3.9$. Show on the right are close-ups of the reconnection layer.

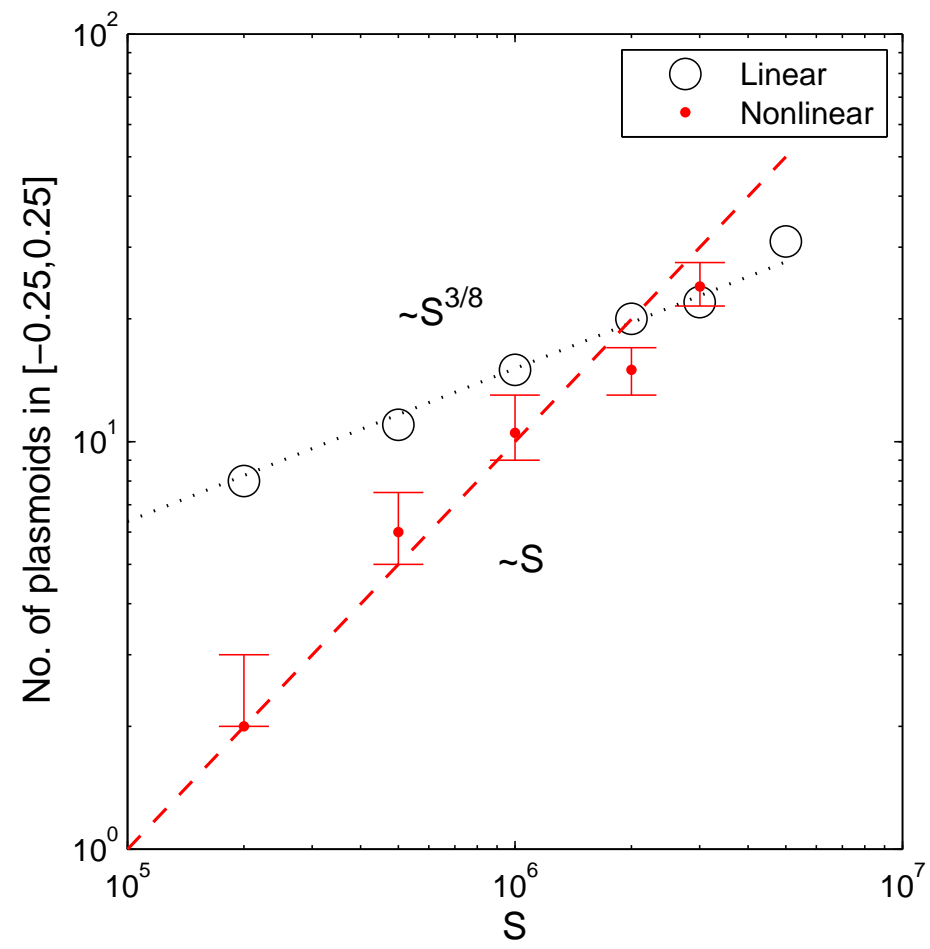

Figure 5: (Color online) Number of plasmoids in the linear and the nonlinear regime. 


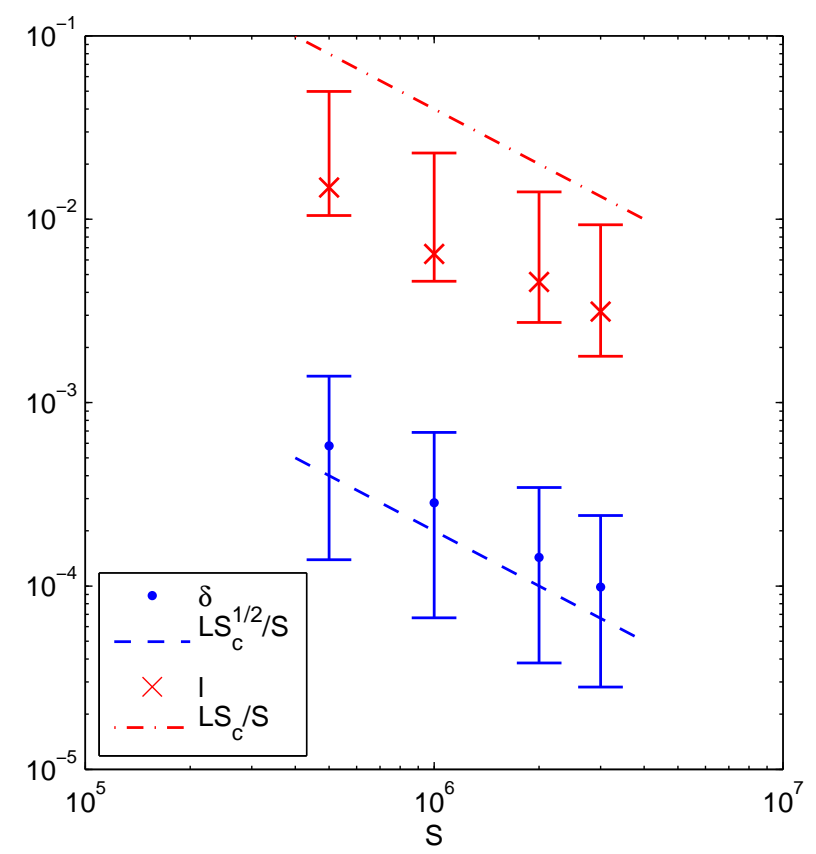

Figure 6: (Color online) Scalings of the current sheet thickness $\delta$ and length $l$ with respect to the global Lundquist number $S$. Also shown for reference are the predictions from the heuristic argument.

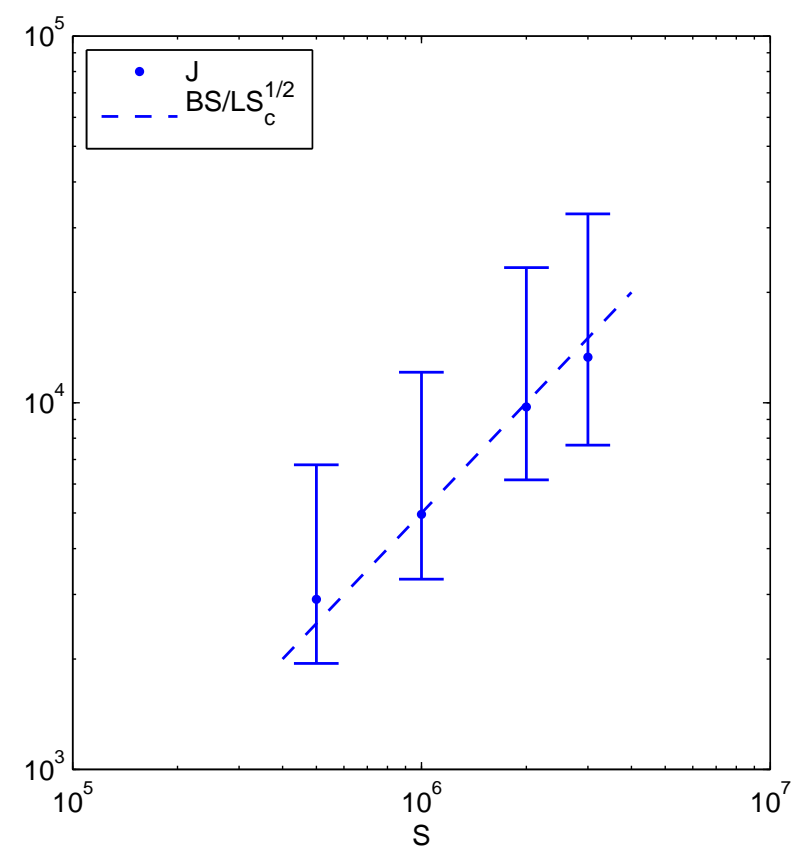

Figure 7: (Color online) The scaling of the local peak current density. Also shown for reference is the prediction from the heuristic argument. 


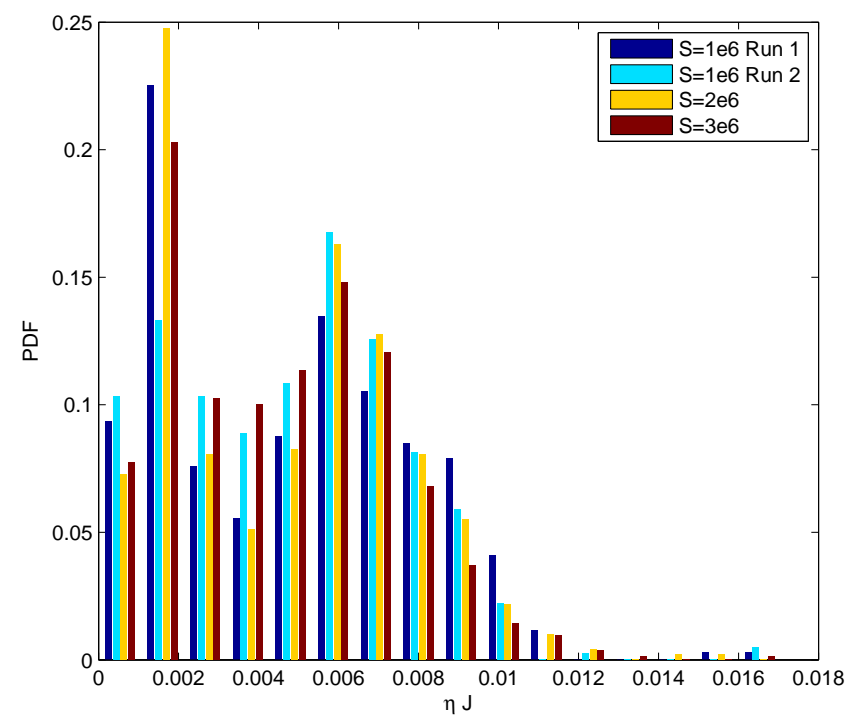

Figure 8: (Color online) The probability distribution function of local $\eta J$ for different $S$, from cases with $\epsilon=10^{-3}$. 